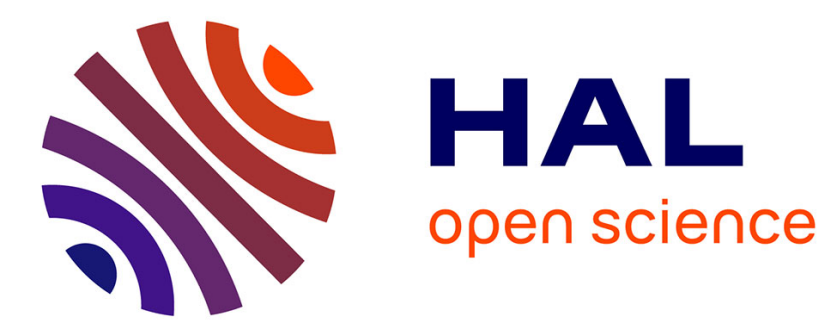

\title{
Effet d'une fumure carbonée sur les capacités d'assimilation nette du radis selon l'éclairement de culture
}

Laurette Combe

\section{To cite this version:}

Laurette Combe. Effet d'une fumure carbonée sur les capacités d'assimilation nette du radis selon l'éclairement de culture. Agronomie, 1981, 1 (2), pp.93-98. hal-00884229

\section{HAL Id: hal-00884229 \\ https://hal.science/hal-00884229}

Submitted on 1 Jan 1981

HAL is a multi-disciplinary open access archive for the deposit and dissemination of scientific research documents, whether they are published or not. The documents may come from teaching and research institutions in France or abroad, or from public or private research centers.
L'archive ouverte pluridisciplinaire HAL, est destinée au dépôt et à la diffusion de documents scientifiques de niveau recherche, publiés ou non, émanant des établissements d'enseignement et de recherche français ou étrangers, des laboratoires publics ou privés. 


\section{Effet d'une fumure carbonée sur les capacités d'assimilation nette du radis selon l'éclairement de culture}

Laurette COMBE

Station de Bioclimatologie, I.N.R.A. Versailles, 78000 Versailles.

\section{RESUME}

Radis,

Fumure carbonée,

Assimilation

nette du $\mathrm{CO}_{2}$,

Eclairement

de culture,

Adaptation.

\begin{abstract}
La fumure carbonée (à $950 \mathrm{vpm}$ de $\mathrm{CO}_{2}$ au lieu de $350 \mathrm{vpm}$, teneur normale de l'air en $\mathrm{CO}_{2}$ ) ne modifie pas les capacités d'assimilation nette de radis cultivés sous fort éclairement $\left(650 \mu \mathrm{E} \mathrm{m}^{-2} \mathrm{~s}^{-1}\right)$. Par contre, les capacités d'assimilation nette de radis cultivés sous faible éclairement $\left(110 \mu \mathrm{E} \mathrm{m}^{-2} \mathrm{~s}^{-1}\right)$ sont diminuées par l'application prolongée de la fumure carbonée.

La valeur du taux d'assimilation nette à $350 \mathrm{vpm}$ de radis cultivés à $350 \mathrm{vpm}$ sous faible éclairement est sensiblement la même que la valeur du taux d'assimilation nette à $950 \mathrm{vpm}$ de Radis cultivés à $950 \mathrm{vpm}$ sous faible éclairement.

Entre 15 et 22 jours, il n'y a pas d'effet de l'âge sur les capacités de photosynthèse des radis quelles que
\end{abstract} soient les conditions de culture (éclairement et $\mathrm{CO}_{2}$ ).

\section{ABSTRACT \\ Radish, Carbon dioxyde enrichment, Net assimilation of $\mathrm{CO}_{2}$, Cultural Illumination, Adaptation.}

Effect of a carbonaceous supply on the net assimilation power in radish according to culture lighting

\begin{abstract}
Carbonaceous supply (at $950 \mathrm{vpm}$ of $\mathrm{CO}_{2}$ instead of $350 \mathrm{vpm}$ normal content in $\mathrm{CO}_{2}$ of the air) does not change the net assimilation power of radishes cultivated under intensive lighting $\left(650 \mu \mathrm{E} \mathrm{m}^{-2} \mathrm{~s}^{-1}\right)$. On the contrary the net assimilation power of radishes grown under faint lighting $\left(110 \mu \mathrm{E} \mathrm{m}^{-2} \mathrm{~s}^{-1}\right)$ is reduced by a long term supply of carbonaceous manure.

The value of the net assimilation rate at $350 \mathrm{vpm}$ under faint lighting is almost the same as the value of the net assimilation rate at $950 \mathrm{vpm}$ in radishes grown under faint lighting.

Between 15 and 22 days there is no age effect on the photosynthesis capacity of radishes whatever the culture
\end{abstract} conditions may be (lighting and $\mathrm{CO}_{2}$ ).

\section{INTRODUCTION}

Depuis longtemps déjà, l'enrichissement de la teneur en gaz carbonique de l'air (fumure carbonée) est connu comme moyen d'augmenter la production des cultures en serre. RUDD-JONES (1972) rappelle dans un historique sur les milieux contrôlés les déjà nombreux travaux qui montrent l'effet bénéfique de la fumure carbonée sur la croissance de tomates, concombres, laitues, chrysanthèmes, etc...

Cet effet de l'enrichissement en $\mathrm{CO}_{2}$ a été attribué à un effet sur la photosynthèse. En effet, les mesures d'échanges gazeux instantanés au niveau des feuilles montrent que l'augmentation de la teneur en $\mathrm{CO}_{2}$ permet d'accroître l'assimilation nette de nombreuses plantes (effet à court terme) (GAASTRA, 1959 ; CHARTIER, 1966).

Mais peu d'auteurs, en dehors de NEALES \& NiCHOLLS (1978), se sont étonnés de la relative faiblesse de l'effet de la fumure carbonée sur la croissance par rapport à son effet sur l'assimilation nette instantanée. En fait, pour compren- dre les effets de la fumure carbonée sur la croissance des plantes, c'est l'effet à long terme de cette fumure sur l'assimilation photosynthétique qu'il faut étudier. En particulier, il est intéressant de savoir si les plantes cultivées en atmosphère enrichie en $\mathrm{CO}_{2}$ ont la même réponse photosynthétique instantanée aux variations de teneur en $\mathrm{CO}_{2}$ que les plantes restées en atmosphère normale.

Autrement dit, la question se pose de savoir s'il existe un effet d'adaptation à la teneur en $\mathrm{CO}_{2}$ comme il existe un effet d'adaptation à l'éclairement de croissance (PRIOUL, 1971).

Un travail précédent (COMBE, 1979) nous avait laissé entrevoir l'existence d'un effet combiné des conditions d'éclairement et de teneur en $\mathrm{CO}_{2}$ de culture sur l'assimilation nette de Radis cultivés en serre. Nous avons donc conçu une expérience pour étudier les courbes de réponse du taux d'assimilation nette à la teneur en $\mathrm{CO}_{2}, \mathrm{~N}(\mathrm{C})$, pour des plantes cultivées avec ou sans fumure carbonée et sous un éclairement fort ou faible. 


\section{DISPOSITIF EXPERIMENTAL}

\subsection{Matériel végétal}

La plante utilisée est le Radis parce qu'il répond bien à la fumure carbonée tant au niveau de la croissance (KNECHT, 1975) qu'à celui de l'assimilation (NILOVSKAYA, 1968) et aussi parce qu'il pousse vite, ce qui permet de faire des expériences répétées. Pour les besoins de l'expérimentation, la variété utilisée est une variété adaptée à la culture en serre. Il s’agit de la variété Serra (Clause).

\subsection{Conditions de culture}

Les radis sont cultivés en pots sur du sable arrosé de solution nutritive complète (COÏC \& LESAINT, 1975). Les pots sont placés dans des enceintes de culture à atmosphère artificielle (température diurne $19{ }^{\circ} \mathrm{C}$, température nocturne $13^{\circ} \mathrm{C}$ ). La durée de l'éclairement est de $14 \mathrm{~h}$ par jour. Deux niveaux d'éclairement sont appliqués aux plantes: un niveau $\mathrm{E}$, éclairement fort, de $190 \mathrm{Wm}^{-2}$ de rayonnement visible soit $650 \pm 34 \mu \mathrm{E} \mathrm{m}^{-2} \mathrm{~s}^{-1}$. Il est obtenu avec des lampes HQI (OSRAM) aux halogènes métalliques placées à $2 \mathrm{~m}$ au-dessus du sol. Le niveau e, éclairement faible, est de $32 \mathrm{Wm}^{-2}$ (visible) soit $110 \pm 6 \mu \mathrm{E} \mathrm{m}^{-2} \mathrm{~s}^{-1}$. Un grillage plastique noir qui ne laisse passer que 17 p. 100 du rayonnement incident a été interposé entre les lampes et les plantes pour obtenir ce niveau de rayonnement.

Deux niveaux de gaz carbonique sont également appliqués pour chacun des 2 niveaux d'éclairement. Dans une enceinte, l'air est à la teneur c normale de $\mathrm{CO}_{2}$ de $350 \pm 50 \mathrm{vpm}$. Dans l'autre enceinte de culture, l'air est enrichi en gaz carbonique (fumure carbonée) à la teneur $\mathrm{C}$ de $950 \pm 50 \mathrm{vpm}$. La concentration en $\mathrm{CO}_{2}$ de l'air des enceintes est mesurée à l'aide d'un analyseur infra-rouge. Le signal électrique de l'analyseur est numérisé et comparé à une valeur de consigne affichée sur un détecteur de seuil électronique. Celui-ci commande l'injection de $\mathrm{CO}_{2}$ lorsque la teneur de l'air est plus faible que la consigne (le jour) et l'absorption de $\mathrm{CO}_{2}$ lorsque la teneur de l'air est plus éle vée que la consigne (la nuit).

\subsection{Protocole expérimental}

La combinaison des 2 niveaux de $\mathrm{CO}_{2}$ avec les 2 éclairements donne donc 4 milieux différents : Ec, EC, ec, eC. Dans chacun de ces 4 milieux, 8 semis échelonnés ont été effectués simultanément de façon à obtenir 8 répétitions pour chacun des traitements.

Aux $15^{\mathrm{e}}$ et $22^{\mathrm{e}}$ jours après le semis, un pot est prélevé dans chaque milieu pour procéder à des mesures d'assimilation nette en chambre d'assimilation. Comme nous disposons de 4 chambres où il est possible de faire simultanément les mesures, les pots de chacun des 4 traitements sont prélevés en même temps (le matin) et mesurés en même temps.

Les chambres d'assimilation sont du type de celle décrite par CHARTIER \& CHARTIER (1971). Les chambres sont en circuit ouvert. L'air est décarboxylé à l'entrée du circuit par passage sur de la chaux sodée, puis sa teneur en gaz carbonique est réglée manuellement aux niveaux choisis pour l'établissement de la courbe $\mathrm{N}(\mathrm{C})$ par enrichissement à partir d'une bouteille contenant 5 p. 100 de $\mathrm{CO}_{2}$. Par un jeu d'électrovannes, l'air qui entre dans la chambre et l'air qui est à l'intérieur de la chambre, sont analysés successivement par un analyseur infra-rouge absolu. Après 15 à $30 \mathrm{mn}$ de stabilisation pour chaque niveau de mesure, l'air est analysé pendant environ $5 \mathrm{mn}$ à l'entrée puis à la sortie de chaque chambre.

Le pot de radis est introduit en entier dans la chambre d'assimilation. Pot et substrat sont enveloppés d'un film plastique ne laissant dépasser que les feuilles. Il a été vérifié que le dégagement de $\mathrm{CO}$, à partir du sol et des racines (feuilles coupées) est indétectable dans ce dispositif de mesure. Le Radis a 2 ou 3 paires de feuilles disposées en rosette, elles ne se recouvrent pas (en général) les unes par les autres, mais elles ne sont pas horizontales.

Or l'éclairement dans les chambres d'assimilation est mesuré sur un plan horizontal. Le rapport de la surface de projection verticale des plantes (mesurée par photographie en vue de dessus) à la surface réelle planimétrée directement donne le cosinus de l'angle d'inclinaison moyen des feuilles par rapport à l'horizontale. L'éclairement moyen reçu par les feuilles est égal à l'éclairement mesuré sur le plan horizontal multiplié par le cosinus de l'inclinaison des feuilles. Selon leur inclinaison, l'éclairement reçu par les feuilles de Radis pour les courbes $\mathrm{N}(\mathrm{C})$ va de $230 \mathrm{Wm}^{-2}$ (visible) ou $1080 \mu \mathrm{E} \mathrm{m}^{-2} \mathrm{~s}^{-1}$ à $320 \mathrm{Wm}^{-2}$ (visible) ou $1520 \mu \mathrm{E} \mathrm{m}^{-2} \mathrm{~s}^{-1}$. Dans tous les cas, il s'agit d'un éclairement saturant: les courbes sont donc comparables.

Pour chaque milieu de culture, les mesures de $\mathrm{N}(\mathrm{C})$ ont été répétées 8 fois avec des plantes différentes mais de même âge : une étude statistique des paramètres des courbes a été faite pour chaque traitement.

\section{RÉSULTATS}

\subsection{Effet de l'âge}

Comme nous le voyons sur l'exemple de la figure 1, les mesures effectuées avec des radis de $15 \mathrm{j}$. et celles portant sur des radis de $22 \mathrm{j}$. sont peu différentes. Pour les radis du traitement Ec, le taux d'assimilation nette des plantes de $22 \mathrm{j}$. est légèrement supérieur à celui des plantes de $15 \mathrm{j}$., mais seuls les points des courbes moyennes situés entre 350 et $550 \mathrm{vpm}$ sont significativement différents au seuil de 0,05 . Cet effet de l'âge est encore moins marqué pour les autres lots de radis ( $\mathrm{EC}$, ec et $\mathrm{eC}$ ). Par la suite, nous comparerons donc des courbes moyennes obtenues à partir des mesures faites à 15 et $22 \mathrm{j}$., soit 16 répétitions.

\subsection{Comparaison des courbes d'assimilation $\mathrm{N}(\mathrm{C})$ des radis cultivés sous fort éclairement}

La figure 2 montre que les courbes moyennes $\mathrm{N}(\mathrm{C})$ des plantes cultivées sous fort éclairement ne sont pas significativement différentes avec ou sans fumure carbonée: les 2 bandes de confiance pour chaque courbe se chevauchent largement. Les 2 courbes moyennes se recoupent à leurs 2 extrémités ( 0 et $1400 \mathrm{vpm})$. La courbe des plantes avec fumure carbonée a seulement tendance à être un peu plus tendue (plus basse) entre 300 et $700 \mathrm{vpm}$ que celle des plantes sans fumure carbonée (les différences entre les points des courbes moyennes ne sont pas significatives).

Le tableau 1 montre que si les ordonnées à l'origine des courbes $\mathrm{N}(\mathrm{C})$ (respiration dans l'air sans $\mathrm{CO}_{2}$ ) sont identiques pour les 2 traitements à fort éclairement, les pentes à l'origine (conductance totale) sont légèrement différentes (significatif au seuil 0,05 ) et les points de compensation ne sont pas significativement différents.

Ainsi, pour des plantes cultivées sous éclairement fort, une fumure carbonée de $950 \mathrm{vpm}$, sous un éclairement 
$\mathrm{N} \mathrm{\mu gCO}_{2} \mathrm{~m}^{-2}{ }_{\mathrm{s}}^{-1}$

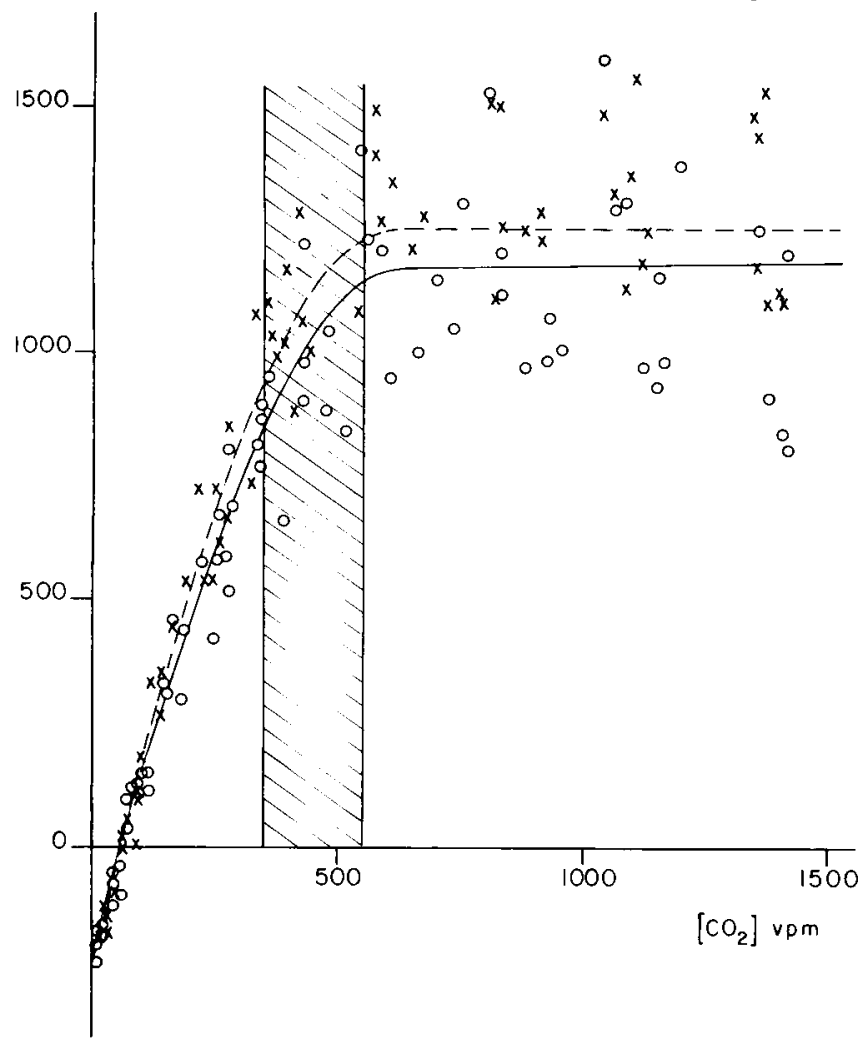

Figure 1

Effet de l'âge sur le taux d'assimilation nette $(N)$ de radis $(E c)$ cultivés à éclairement fort et sans fumure carbonée. Points de mesure et courbes moyennes sur 8 répétitions mesurés à éclairement $E \geqslant 230 \mathrm{Wm}^{-2}$ (visible) ou $1090 \mu E \mathrm{~m}^{-2} \mathrm{~s}^{-1}$.

Effect of age on net assimilation rate $(N)$ of radishes Ec grown under strong irradiance and normal $\mathrm{CO}_{2}$; measured points and mean curves on 8 replicates under irradiance $E \geqslant 230 \mathrm{Wm}^{-2}$ (visible) or $1090 \mu \mathrm{E} \mathrm{m}^{-2} \mathrm{~s}^{-1}$

$0-15$ jours (15 days old radishes)

$x-\ldots 22$ jours ( 22 days old radishes)

Zone où les courbes moyennes sont significativement différentes au seuil 0,05 p. 100.

Area where mean curves are significantly different at the 0,05 level.

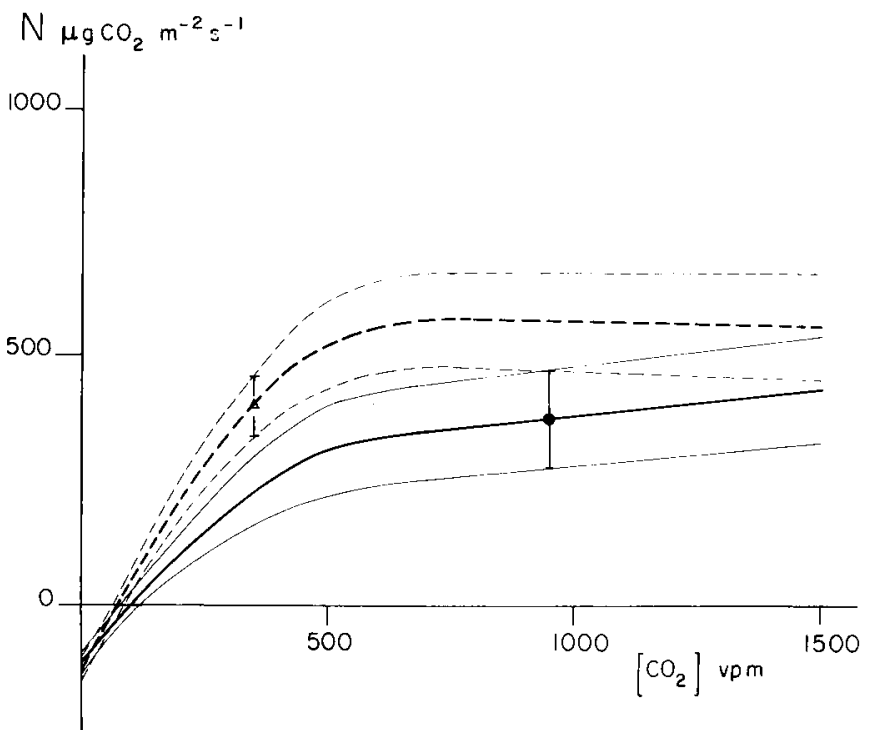

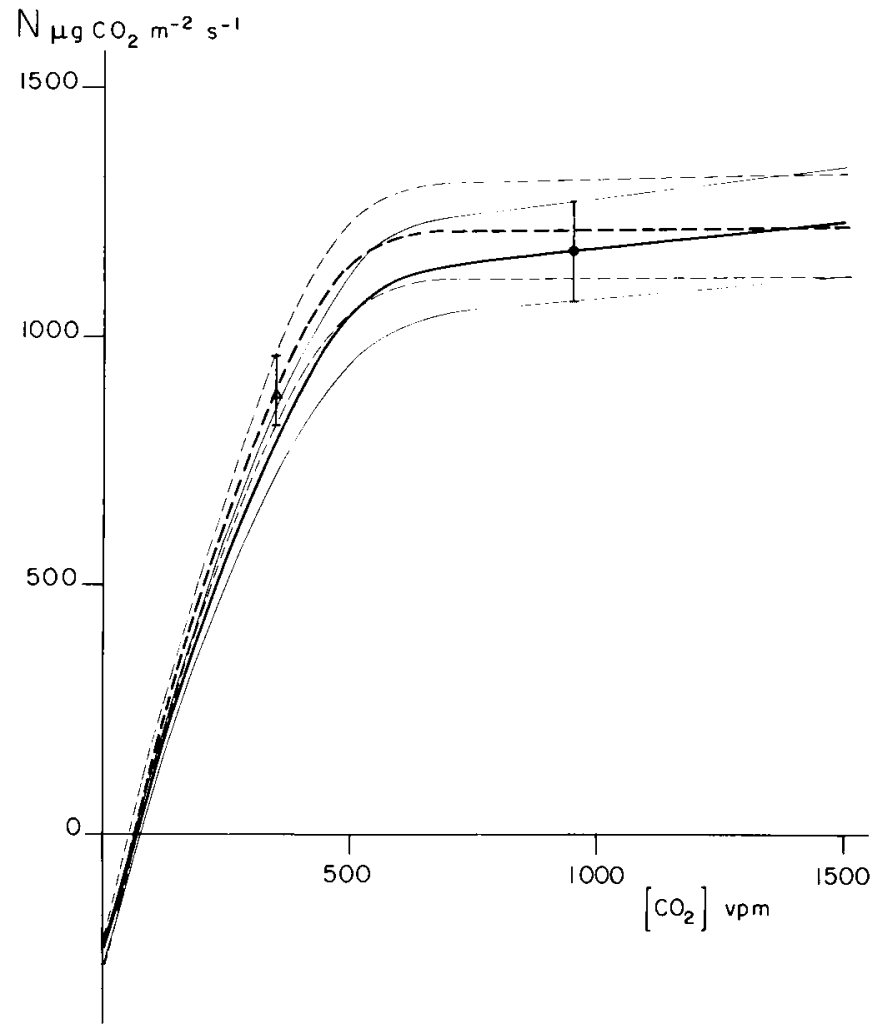

Figure 2

Comparaison des courbes $N(C)$ pour des radis cultivés sous éclairement fort avec $(E C)$ ou sans $(E c)$ fumure carbonée. Ordonnées: taux d'assimilation nette par unité de surface de feuille. (16 répétitions).

$N(C)$ curves for radishes grown under strong irradiance with or without high $\mathrm{CO}_{2}$ (16 replicates). Ordinate: net assimilation rate.

EC courbe moyenne (mean curve).

Ec courbe moyenne (mean curve).

Bande de confiance au seuil de 5 p. 100 pour EC.

Confidence belt at the 0,05 level for $\mathrm{EC}$.

Bande de confiance au seuil de 5 p. 100 pour Ec.

Confidence belt at the 0,05 level for $\mathrm{Ec}$.

\section{Figure 3}

Comparaison des courbes $N(C)$ pour des radis cultivés sous faible éclairement avec (eC) ou sans (ec) fumure carbonée. Ordonnées: taux d'assimilation nette par unité de surface de feuille. (16 répétitions).

$N(C)$ curves for radishes grown under poor irradiance with or without high $\mathrm{CO}_{2}$ (16 replicates). Ordinate : net assimilation rate.

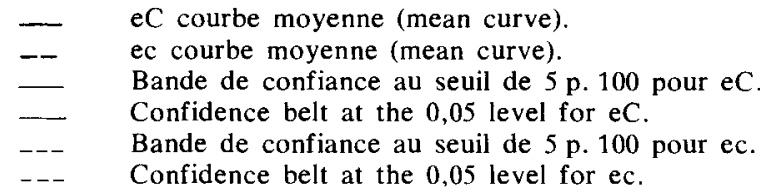


TABLEAU 1

Comparaison des paramètres des courbes $N(C)$ pour les 4 traitements (moyenne sur 16 répétitions) (les mesures ont été effectuées en éclairement saturant).

Parameters of $N(C)$ curves for the 4 treatments (16 replicates); measures were taken under saturating illumination.

\begin{tabular}{|c|c|c|c|c|c|c|}
\hline & \multicolumn{3}{|c|}{$\begin{array}{l}\text { Eclairement de la culture fort } \\
\text { (cultural illumination strong) }\end{array}$} & \multicolumn{3}{|c|}{$\begin{array}{l}\text { Eclairement de la culture faible } \\
\text { (cuitural illumination weak) }\end{array}$} \\
\hline & Ec & & $\mathrm{EC}$ & ec & & $\mathrm{eC}$ \\
\hline $\begin{array}{l}\text { Respiration dans l'air sans } \mathrm{CO}_{2}\left(\mu \mathrm{g} \mathrm{CO}_{2} \mathrm{~m}^{-2} \mathrm{~s}^{-1}\right) \\
\left.\text { (respiration in air without } \mathrm{CO}_{2}\right)\end{array}$ & -243 & ns & -244 & -132 & ns & -114 \\
\hline $\begin{array}{l}\text { Conductance totale }\left(10^{-3} \mathrm{~m} \mathrm{~s}^{-1}\right) \text { (pente à l'origine) } \\
\text { (total conductance) }\end{array}$ & 2,06 & & 1,89 & 0,97 & $* * *$ & 0,61 \\
\hline $\begin{array}{l}\text { Point de compensation } \Gamma\left(\mathrm{vpm} \text { de } \mathrm{CO}_{2}\right) \\
\text { (compensation point) }\end{array}$ & 61 & ns & 67 & 73 & * & 98 \\
\hline $\begin{array}{l}\text { Taux d'assimilation nette à } 250 \mathrm{vpm}\left(\mu \mathrm{CO}_{2} \mathrm{~m}^{-2} \mathrm{~s}^{-8}\right) \\
\text { (net assimilation rate at } 350 \mathrm{vpm})\end{array}$ & 887 & $*$ & 786 & 397 & $* * *$ & 227 \\
\hline $\begin{array}{l}\text { Taux d'assimilation nette à } 1000 \mathrm{vpm}\left(\mu \mathrm{gCO}_{2} \mathrm{~m}^{-2} \mathrm{~s}^{-1}\right) \\
\text { (net assimilation rate at } 1000 \mathrm{vpm})\end{array}$ & 1215 & $\mathrm{~ns}$ & 1176 & 569 & $* *$ & 381 \\
\hline $\begin{array}{l}\text { ns différence non significative entre les plantes avec } \\
* \text { différence significative au seuil } 0,05 \text {. } \\
\text { ** différence significative au seuil } 0,01 \text {. } \\
\text { *** différence significative au seuil } 0,001 \text {. }\end{array}$ & sans $f$ & & & & & \\
\hline
\end{tabular}

saturant $\left(E>190 \mathrm{Wm}^{-2}\right.$ (visible)) auquel correspondent les courbes de la figure 2 , fait passer le taux d'assimilation nette de 887 à $1176 \mu \mathrm{g} \mathrm{CO}_{2} \mathrm{~m}^{-2}$ (de feuille) s ${ }^{-1}$ (voir tabl. 1), ce qui correspond à une augmentation de 33 p. 100 (différence significative au seuil de 0,001 ).

\subsection{Comparaison des courbes d'assimilation $\mathrm{N}(\mathrm{C})$ des radis cultivés sous faible éclairement}

La figure 3 montre que les courbes moyennes $N(C)$ des plantes avec et sans fumure carbonée sous faible éclairement sont significativement différentes. Les 2 courbes moyennes ne se recoupent pas et les bandes de confiance ne se chevauchent qu'aux 2 extrémités.

La fumure carbonée déprime nettement la courbe de photosynthèse $\mathrm{N}(\mathrm{C})$ mesurée sous éclairement fort lorsque l'éclairement de culture est faible. La courbe $\mathrm{N}(\mathrm{C})$ a été mesurée une fois pour chaque traitement avec un éclairement faible (de 28 à $35 \mathrm{Wm}^{-2}$ (visible) ou 133 à $166 \mu \mathrm{E} \mathrm{m}^{-2} \mathrm{~s}^{-1}$ ) de façon à savoir ce qui se passe réellement dans les conditions de croissance. Sur l'exemple de la figure 4, nous voyons que l'effet dépressif de la fumure carbonée sur la courbe $\mathrm{N}(\mathrm{C})$ mis en évidence pour des mesures faites à fort éclairement se manifeste de la même façon pour des mesures faites à faible éclairement.

Nous voyons sur le tableau 1 que, pour les plantes cultivées à faible éclairement, la différence entre les ordonnées à l'origine des courbes $\mathrm{N}(\mathrm{C})$ n'est pas significative ; par contre, la pente à l'origine est significativement différente (seuil de 0,001 ) et le point de compensation également (seuil de 0,05 ). Sous éclairement faible, la fumure carbonée ne permet donc pas de gain de photosynthèse ; au contraire même, l'assimilation nette à $950 \mathrm{vpm}$ de plantes traitées par une forte teneur en $\mathrm{CO}_{2}$ a tendance à être plus faible que l'assimilation nette à $350 \mathrm{vpm}$ de plantes sans fumure carbonée (différence non significative) (voir tabl. 1).

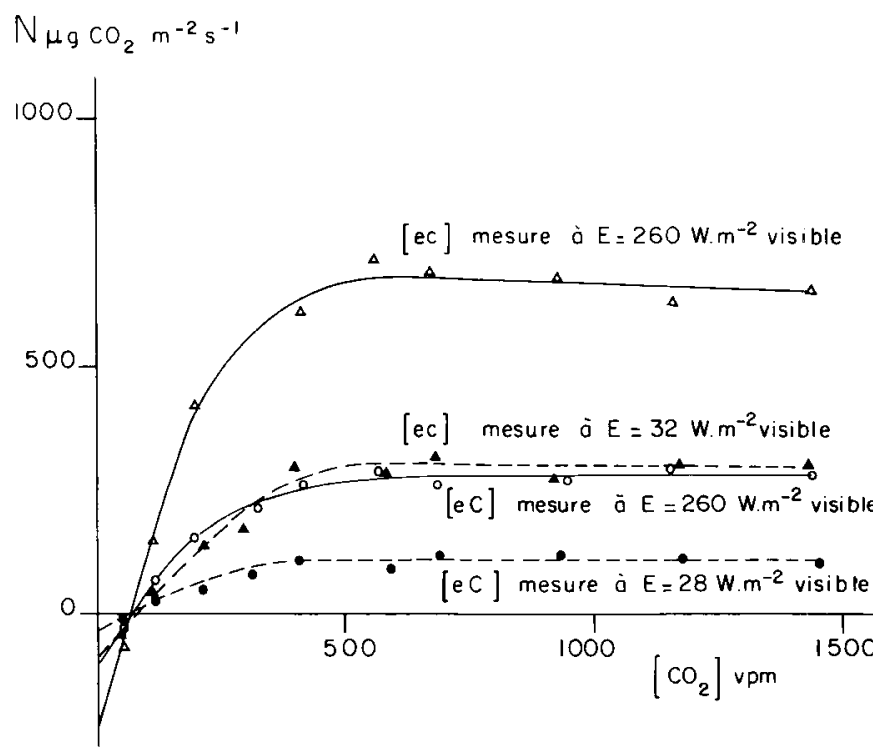

Figure 4

Comparaison des courbes $N(C)$ établies à éclairement fort (260 Wm-2 visible) avec les courbes établies à faible éclairement $\left(\simeq 30 \mathrm{Wm}^{-2}\right.$ visible $)$ pour des radis de 22 jours cultivés sous faible éclairement avec $(e C)$ ou sans fumure carbonée (ec).

$N(C)$ curves measured at strong irradiance $\left(260 \mathrm{Wm}^{-2}\right.$ visible) and curves measured at poor irradiance $\left(\simeq 30 \mathrm{Wm}^{-2}\right.$ visible) for 22 days old radishes grown under poor irradiance with or without high $\mathrm{CO}_{2}$.

\section{DISCUSSION ET CONCLUSION}

Nous voyons que chez le Radis, l'effet de la fumure carbonée à long terme (ou effet adaptatif) sur le taux d'assimilation nette mesuré en éclairement saturant est $\therefore$ ':fférent selon l'éclairement de la culture. Lorsque l'éclaire- 
ment moyen de croissance est suffisamment élevé (ici $190 \mathrm{Wm}^{-2}$ visible ou $650 \mu \mathrm{E} \mathrm{m}^{-2} \mathrm{~s}^{-1}$ ), la fumure carbonée ne modifie que fort peu les courbes d'assimilation en fonction de la teneur en $\mathrm{CO}_{2}$. Dans ce cas, la fumure carbonée à $950 \mathrm{vpm}$ permet un gain de photosynthèse qui se traduit par une croissance plus forte en matière sèche (COMBE, 1979).

Par contre, lorsque l'éclairement de la culture est faible (ici $32 \mathrm{Wm}^{-2}$ (visible) ou $110 \mu \mathrm{E} \mathrm{m}^{-2} \mathrm{~s}^{-1}$ ), la fumure carbonée entraîne une diminution de la capacité photosynthétique (mesurée en lumière saturante) des radis pour les différentes concentrations de $\mathrm{CO}_{2}$ (de 0 à $1400 \mathrm{vpm}$ ).

Cette interaction de la fumure carbonée avec un autre paramètre de l'assimilation (ici l'éclairement) sur l'effet adaptatif $\mathrm{du} \mathrm{CO}_{2}$ se rencontre aussi pour d'autres facteurs. Par exemple pour la température, HOFSTRA \& HESKETH (1975) constatent que la fumure carbonée a un effet positif sur la croissance du Soja uniquement lorsque les températures de la culture se situent à l'optimum (pour la croissance à la teneur normale de $\mathrm{CO}_{2}$ ).

De même, l'âge de la plante peut interférer avec la réaction au gaz carbonique. NEALES \& NiCHOLLS (1978) constatent que le taux d'assimilation nette est augmenté en passant de 200 à $800 \mathrm{vpm}$ de $\mathrm{CO}_{2}$ pour des blés de $10 \mathrm{j}$. alors qu'il est diminué pour des blés de $24 \mathrm{j}$. dans les mêmes conditions. Il peut s'agir là d'un effet de la durée d'application du traitement de fumure carbonée plutôt que d'un effet de l'âge proprement dit. En tous cas, chez le Radis, nous ne constatons pas une telle différence entre 15 et 22 jours.

L'interaction éclairement-gaz carbonique que nous avons mise en évidence chez le Radis se rencontre pour d'autres plantes. Pour la Laitue, par exemple, ROBELIN \& MARTIGNAC (1975) montrent qu'à des éclairement élevés un effet dépressif des teneurs en $\mathrm{CO}_{2}$ supérieures à $1300 \mathrm{vpm}$ se manifeste plus nettement qu'à des éclairements faibles.
Inversement, GIFFORD (1977) constate que le gain de croissance dû à une fumure carbonée est relativement plus fort pour du blé cultivé sous un éclairement faible que sous un éclairement élevé, cependant il n'obtient pas de différence significative entre les courbes $\mathrm{N}(\mathrm{C})$ des plantes cultivées à 140, 290 ou $490 \mathrm{vpm}$ de $\mathrm{CO}_{2}$ sous éclairement faible (lumière d'hiver).

L'action du gaz carbonique sur l'ou verture stomatique est considérée comme une explication possible de l'effet dépressif de la fumure carbonée sur la capacité d'assimilation (cas du chou-rave, FRYDRYCH, 1978). Chez le Radis, nous avons fait des mesures de résistance stomatique dont les résultats sont résumés dans le tableau 2. Effectivement, l'augmentation de la teneur en $\mathrm{CO}_{2}$ de 350 à $1000 \mathrm{vpm}$ accroît la résistance stomatique, surtout si la plante a reçu une fumure carbonée et un éclairement faible. Mais la part de la résistance stomatique dans la résistance totale à la diffusion du $\mathrm{CO}_{2}$ reste faible dans tous les traitements $\left(\mathrm{r}_{\mathrm{s}} / \sum \mathrm{r}=18 \mathrm{p} .100\right.$ pour les plantes avec $\mathrm{E}$ et $r_{s} / \sum r=9$ p. 100 pour les plantes avec e). La fumure carbonée accroît donc beaucoup plus la résistance interne que la résistance stomatique.

L'effet dépressif de l'application prolongée de la fumure carbonée sur le taux d'assimilation nette des radis élevés en lumière faible se rencontre pour d'autres plantes. FRYDRYCH (1976) le voit chez de jeunes concombres (fumure carbonée à $1500 \mathrm{vpm}$, éclairement de la culture sous $35 \mathrm{Wm}^{-2}$ ). MADSEN (1975) constate le même phénomène chez la Tomate adulte (fumure carbonée à $2209 \mathrm{vpm}$ ). Enfin Ho (1977) retrouve ce résultat aussi chez la Tomate (fumure carbonée à $1000 \mathrm{vpm}$, éclairement $40 \mathrm{Wm}^{-2}$ ). Cette diminution du taux de photosynthèse chez les plantes enrichies en $\mathrm{CO}_{2}$ est attribuée par la plupart des auteurs à une mauvaise évacuation des assimilats hors des feuilles et à une augmentation du contenu en amidon des chloroplastes.

\section{TABLEAU 2}

Comparaison des résistances à la diffusion du $\mathrm{CO}_{2}$ pour les 4 traitements

Radis de 22 jours, mesures effectuées en éclairement saturant.

Valeurs moyennes \pm écart-type de la moyenne (8 répétitions).

Résistance de la couche limite $r_{a}=55 \pm 3 \mathrm{sm}^{-1}$.

Résistance interne $r_{i}=\sum r-\left(r_{a}+r_{s}\right)$.

Resistance to $\mathrm{CO}_{2}$ diffusion for the 4 treatments.

22 days old radishes, measures taken under saturating illumination.

Mean values \pm standart deviation (8 replicates).

boundary layer resistance $r_{a}=55 \pm 3 \mathrm{sm}^{-1}$.

Internal resistance $r_{i}=\sum r-\left(r_{a}+r_{s}\right)$

\begin{tabular}{|c|c|c|c|c|}
\hline \multirow{2}{*}{ Résistances $\mathrm{sm}^{-1}$} & \multicolumn{4}{|c|}{ Traitements } \\
\hline & Ec & $\mathrm{EC}$ & ec & $\mathrm{eC}$ \\
\hline $\begin{array}{l}\mathrm{r}_{\mathrm{s}}(350) \text { résistance stomatique à } 350 \mathrm{vpm} \mathrm{CO}_{2} \\
\text { (stomatal resistance at } 350 \mathrm{vpm} \text { ) } \\
\mathrm{r}_{\mathrm{s}}(1000) \text { résistance stomatique à } 1000 \mathrm{vpm} \mathrm{CO}_{2} \\
\text { (stomatal resistance at } 1000 \mathrm{vpm})\end{array}$ & $\begin{array}{l}87 \pm 16 \\
+9 \% \mathrm{~ns} \\
95 \pm 11\end{array}$ & $\begin{array}{r}69 \pm 13 \\
+33 \% * \\
92 \pm 20\end{array}$ & $\begin{array}{r}90 \pm 7 \\
+21 \% * \\
109 \pm 9\end{array}$ & $\begin{array}{r}107+17 \\
+39 \% * \\
149 \pm 21\end{array}$ \\
\hline $\begin{array}{l}\sum \mathrm{r} \text { résistance totale (d'après la pente à l'origine) } \\
\text { (total resistance (from initial slope)) }\end{array}$ & $485 \pm 8$ & $528 \pm 10$ & $1031 \pm 36$ & $1639 \pm 9$ \\
\hline$r_{i}(350) / \sum r$ & 0,71 & 0,77 & 0,86 & 0,90 \\
\hline$r_{i}(1000) / \sum r$ & 0,69 & 0,72 & 0,84 & 0,88 \\
\hline \multicolumn{5}{|c|}{$\begin{array}{l}\text { ns différence entre les } 2 \text { lignes non significative. } \\
* \text { différence entre les } 2 \text { lignes significative au seuil } 0,05 \text {. } \\
* \text { différence entre les } 2 \text { lignes non significative au seuil } 0,01 \text {. }\end{array}$} \\
\hline
\end{tabular}


Cependant, Ho (1977) constate que les plantes enrichies en $\mathrm{CO}_{2}$ montrent un taux d'exportation hors des feuilles plus élevé que les plantes cultivées en atmosphère normale. De plus, les éclairements de culture (de 30 à $40 \mathrm{Wm}^{-2}$ ) sont loin d'être saturants et l'on voit mal pourquoi l'augmentation de la production d'assimilat par le $\mathrm{CO}_{2}$ provoquerait à elle seule un engorgement du système de transport (qui n'a pas lieu, semble-t-il, quand l'assimilation est augmentée par l'éclairement).

La fumure carbonée provoque donc chez le Radis une augmentation des résistances internes à la diffusion du $\mathrm{CO}_{2}$ lorsqu'elle est appliquée sur des plantes peu éclairées, mais le mécanisme de cette augmentation des résistances internes n'est pas évident.

\section{REMERCIEMENTS}

L'auteur tient à remercier M. André KoBILINSKy pour son appui dans le domaine du dépouillement statistique des courbes d'assimilation $\mathrm{N}(\mathrm{C})$ et $\mathrm{M}$. Bernard JAUSSELY pour son aide technique

Reçu le 23 mai 1980 Accepté le 29 octobre 1980

\section{RÉFÉRENCES BIBLIOGRAPHIQUES}

Chartier P., 1966. Etude théorique de l'assimilation brute de la feuille. Ann. Physiol. Vég., 8, 3, 167-196.

Chartier M., Chartier Ph., 1971. Design of an air conditioned assimilation chamber for detached leaves. Photosynthetica, 5, 1 , 74-75.

Coic Y., Lesaint C., 1975. La nutrition minérale et en eau des plantes en horticulture avancée. Doc. Techn. SCPA, 23, $22 \mathrm{p}$.

Combe L., 1979. Effet du gaz carbonique et de l'éclairement sur la croissance et la répartition des assimilats chez le Radis (Raphanus sativus). Ann. agron., 30, 3, 217-231.

Frydrych J., 1976. Photosynthetic characteristics of cucumber seedlings grown under two levels of carbon dioxide. Photosynthetica, 10, 3, 335-338.

Frydrych J., 1978. The effect of increased $\mathrm{CO}_{2}$ concentration on photosynthetic rate of leaves of kohlrabi clones (Brassica oleracea var. gongyclodes L.). Z. Pflanzenzüchtg., 80, 158-161.

Gaastra P., 1959. Photosynthesis of crop plants as influenced by light, carbon dioxide, temperature, and stomatal diffusion resistance. Meded. Landbouwhogesch. Wageningen, 59, 13, 1-68.

Gifford R. M., 1977. Growth pattern, carbon dioxide exchange and dry weight distribution in wheat growing under differing photosynthetic environments: Aust. J. Plant Physiol., 4, 99-110.

Ho L. C., 1977. Effects of $\mathrm{CO}_{2}$ enrichment on the rates of photosynthesis and translocation of tomato leaves. Ann. Appl. Biol., 87, 191-200.

Hofstra G., Hesketh J. D., 1975. The effects of temperature and $\mathrm{CO}_{2}$ enrichment on photosynthesis in soybean. In : "Environmental and biological control of photosynthesis", Marcelle R. Ed. Junk, The Hague, 71-80.
Knecht G. N., 1975. Response of radish to high $\mathrm{CO}_{2}$. Hortscience, 10, 3, 274-275.

Madsen E., 1975. Effect of $\mathrm{CO}_{2}$ enrichment on growth, development, fruit production and fruit quality of tomato, from a physiological view point. In: “Phytotronics in agriculturan and horticultural research" Phytotronics I. Chouard P. \& de Bilderling N. Ed., Gauthier-Villars, Paris, 318-330.

Neales T. F., Nicholls A. O., 1978. Growth responses of young wheat plants to a range of ambient $\mathrm{CO}_{2}$ levels. Aust. J. Plant Physiol., 5, 45-59.

Nilovskaya N. T., 1968. Photosynthesis and respiration in vegetable plants exposed to various carbon dioxide concentrations. Soviet Plant Physiol., 15, 853-858.

Prioul J. L., 1971. Réaction des feuilles de Lolium multiflorum à l'éclairement pendant la croissance et variation des résistances aux échanges gazeux photosynthétiques. Photosynthetica, 5, 4, 364-375.

Raper P. D., Peedin G. F., 1978. Photosynthetic rate during steady state growth as influenced by carbon dioxide concentration. Bot. Gaz., 139, 2, 147-149.

Robelin M., Martignac M., 1975. Contribution à l'étude de la fumure carbonique sous serre. In: "Phytotronics in agricultural and horticultural research", Phytotronics I, Chouard P., de Bilderling N. Ed., Gauthier-Villars, Paris, 305-317.

Rudd-Jones D., 1972. Controlled environments. In : “Crop processes in controlled environments", Rees A. R. \& al. Ed. Academic Press, London 1-6. 